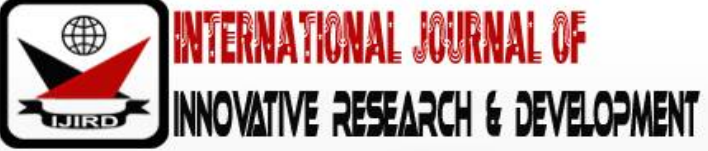

ISSN 2278 - 0211 (Online)

\section{Economic Imperative of Flow Optimization in Natural Gas Pipeline Netw ork System}

\author{
0. M. O. Etebu \\ Professor, Department of Mechanical Engineering, \\ University of Port Harcourt, Port Harcourt, Rivers State, Nigeria \\ Dr. Mathew, Shadrack Uzoma \\ Senior Lecturer, Department of Mechanical Engineering \\ University of Port Harcourt, Port Harcourt, Rivers State, Nigeria
}

\begin{abstract}
:
Economic evaluation of gas pipelines assets and facilities are three-fold in nature. Design, construction and operation costs are the three aspects of the economic evaluation implications. Installation cost of a gas pipeline network system is pressure and flow throughput dependent. The work considers single phase flow of gas in gas pipeline network system. The economic assessment of capital cost savings accruable operating gas pipeline at optimal level of performance considers five operating gas pipelines network system in Nigerian terrain. The case study Natural gas pipelines are ElfTotal Nig. Ltd, Shell Petroleum Development Company (SPDC), Agip Nig. Ltd, Nigeria Gas Company (NGC)-Eastern Division and Nigeria Gas Company NGC)Western Division.

The economic viability or feasibility of a facility is dependent on the cost of development, operation and service life. The consideration also applies to the capital cost savings that could accrue if gas pipeline is designed and operated at their optimal capacities condition.

The economic evaluation results for optimized pipelines operated on Panhandle-A and Modified Panhandle-B were remarkable. Potential capital cost savings for Elf Total optimized Panhandle-A and optimized Modified Panhandle-B was within a margin of 3\% to 31\%. That of Shell Petroleum Development Company (SPGC) was within 5\% to 40\%. Agip Nig. Ltd was to the tune of 3\% TO 44\%. Nigeria Gas Company (NGC) Eastern Division potential savings in installation cost is within $5 \%$ to $36 \%$. Nigeria Gas Company (NGC) Western Division potential savings in investment cost is in the range of $5 \%$ to $56 \%$.
\end{abstract}

Keywords: Economic evaluation; installation cost, optimal, performance; economic viability; service life; optimized pipelines

\section{Introduction}

The world energy supply and consumption scenario over the past couple of centuries confirm that fossil fuels are the primary sources of energy. The rate and level of energy consumption are indications of the level of social, economic and technological growth of any country [1]. Petroleum fuel and its derivatives have superseded the position of coal as the world dominant source of energy in the past decades.

In recent years, continual increase in the price of oil coupled with significant decline in reserve as well as new environmental concerns expressed by various nations of the world about the existing high level of air pollution have led to the exploitation of a cleaner and more economically attractive fuel, such as natural gas.

In relation to petroleum and coal, natural gas is a primary source of energy that is eco-friendlier due to lower carbon dioxide and nitrogen oxide emissions. These gases are known as greenhouse gases with the tendency of increasing the temperature of the earth [2].

As stated by Shell, a global group of energy and petrochemical companies, in a special advertorial in 2000 , the world is hungry for energy. As a

consequence of this demand, a significant effort to increase world energy reserve has been made in the last few decades, despite the recessions and economic issues currently faced by worldwide gas producers $[3,4,5]$.

Current gas reserves in Nigeria are conservatively put at approximately 185 trillion standard cubic feet [6]. Therefore, it is imperative that gas facilities be designed and operated efficiently so that available resources could be conserved and deployed for strategic development of the nation's vast gas resources.

The optimization results obtained from the five gas pipelines used for case study [7,8]. The pipelines are Elf Total Nig. Ltd, Shell Petroleum Development Company (SPDC) Nig. Ltd, Agip Nig. Ltd, Nigeria Gas Company Ltd (Eastern Division) and Nigeria Gas Company Ltd (Western Division). The operating parameters for these pipelines are as presented in Tables 1 and 2.

The economic viability or feasibility of a facility is dependent on the cost of development, operation and service life. These considerations also apply to capital cost savings that could accrue if gas pipelines are designed and operated at 
their optimal capacities condition. According to Abam [9], empirical relationship exists between the capacity (size) of a facility and the associated capital cost or investment cost. The economic imperatives are examined forthe gas pipelines covered in this work on which optimization was carried out.

\subsection{Significance of Study}

Gas pipeline assets and facilities are expensive production assets. The empirical cost estimating scheme has exposed the gains accruable while operating optimally. The savings accruable while operating optimally could be diverted to exploration and development of new gas wells.

\subsection{Relevant Model for Economic Evaluation}

The model for economic assessment is based on scaling law. It is expressed as:

$\frac{\operatorname{COST} A}{\operatorname{COST} B}=\left[\frac{\operatorname{SIZE} A}{\operatorname{SIZE} B}\right]^{N}$

Where:

Cost A-Capital cost of new facility being proposed

Cost B-Capital cost of existing similar facility

Size A-capacity of facility A

Size B-capacity of facility B

$\mathrm{N}-$ empirical exponent, whose value is between 0.4 and 0.8 ; for facilities laid in swamp terrains, $\mathrm{N}=0.6$. This is also applicable to Oil and Gas facilities and most investments in the Petroleum Industry.

The cost comparison is analyzed as follows:

CAPACITY $A=$ Size $A=Q_{o p t}$

CAPACITY B=Size $B=Q_{\text {opr }}$

$\mathrm{Q}_{\text {opt}}$ - optimal flow capacity

Qopr-operational flow capacity

Percentage cost saving is defined as:

$$
\begin{aligned}
100 \times\left(\frac{\operatorname{Cost} A-\operatorname{Cost} B}{\operatorname{Cos} t B}\right) & =100 \times\left(\frac{\operatorname{Cost} A}{\operatorname{Cost} B}-1\right) \\
& =100 \times\left(\left[\frac{\text { Size } A}{\text { Size } B}\right]^{N}-1\right) \\
& =100 \times\left(\left[\frac{Q_{\text {opt }}}{Q_{\text {opr }}}\right]^{N}-1\right) \ldots
\end{aligned}
$$

\subsection{Empirical Model Input Data}

The input data to the computer simulated algorithms [7,8] for the determination of the optimal flow capacities for the case study company are as in Tables 1 and Tables 2. 
ElfTotal Nig. Ltd

Physical Configuration: From Obite to Bonny NLNG

Terrain transversed: From Obite to Ndele to Bonny NLNG

Design Standard Code: ANSI/ASM E B31.8 Standard Code

\begin{tabular}{|c|c|c|c|c|c|c|}
\hline Length $(\mathrm{km})$ & Diameter(cm) & Manifolds & $\begin{array}{l}\text { Design } \\
\text { Pressure(bar) }\end{array}$ & $\begin{array}{l}\text { Input/Output } \\
\text { Pressure(bar) }\end{array}$ & Flow Rate $\left(\mathrm{m}^{3} / \mathrm{s}\right.$ & $\begin{array}{l}\text { Operating } \\
\text { Temperature } \\
\left({ }^{(0} \mathrm{C}\right)\end{array}$ \\
\hline 134 & $36 "(91.44 \mathrm{~cm})$ & 2 & 100 & $84 / 63$ & 1.8 & 40 \\
\hline $\begin{array}{l}\text { Allowable } \\
\text { Pressure } \\
\text { Drop(bar) }\end{array}$ & $\begin{array}{l}\text { Coated/ } \\
\text { Uncoated }\end{array}$ & $\begin{array}{l}\text { Flow } \\
\text { Reynolds } \\
\text { Number }\end{array}$ & Specific Gravity & Buried/Surface & Compressibility Factor & \\
\hline 20 & coated & 4000 & 0.6657 & Buried & 0.749 & \\
\hline
\end{tabular}

Shell Petroleum Development Company

Physical Configuration: From Soku to Bonny NLNG

Terrain transversed: From Soku to Ndele to Bonny NLNG

Design Standard Code: ANSI/ASM E B31.8 Standard Code

\begin{tabular}{|l|l|l|l|l|l|l|}
\hline Length(km) & Diameter(cm) & Manifolds & $\begin{array}{l}\text { Design } \\
\text { Pressure(bar) }\end{array}$ & $\begin{array}{l}\text { Input/Output } \\
\text { Pressure(bar) }\end{array}$ & $\begin{array}{l}\text { Flow Rate }\left(\mathrm{m}^{3} / \mathrm{s}\right. \\
\text { Temperature } \\
\left({ }^{\circ} \mathrm{C}\right)\end{array}$ \\
\hline 116 & $36 "(91.44 \mathrm{~cm})$ & 1 & 100 & $81 / 63$ & 1.8 & 40 \\
\hline $\begin{array}{l}\text { Allowable } \\
\text { Pressure } \\
\text { Drop(bar) }\end{array}$ & Coated/ & $\begin{array}{l}\text { Flow } \\
\text { Reynolds } \\
\text { Number }\end{array}$ & Specific Gravity & Buried/Surface & Compressibility Factor & \\
\hline 20 & coated & 4000 & 0.6978 & Buried & 1.273 \\
\hline
\end{tabular}

\section{Agipl Nig. Ltd}

Physical Configuration: From Obiafu to Bonny NLNG

Terrain transversed: From Obiafu to Bonny NLNG

Design Standard Code: ANSI/ASM E B31.8 Standard Code

\begin{tabular}{|l|l|l|l|l|l|l|}
\hline Length(km) & Diameter(cm) & Manifolds & $\begin{array}{l}\text { Design } \\
\text { Pressure(bar) }\end{array}$ & $\begin{array}{l}\text { Input/Output } \\
\text { Pressure(bar) }\end{array}$ & $\begin{array}{l}\text { Oplow Rate }\left(\mathrm{m}^{3} / \mathrm{s}\right. \\
\text { Temperating } \\
\left({ }^{(0} \mathrm{C}\right)\end{array}$ & \\
\hline 134 & $36^{\prime \prime}(91.44 \mathrm{~cm})$ & 2 & 100 & $84 / 63$ & 1.8 & \\
\hline $\begin{array}{l}\text { Allowable } \\
\text { Pressure } \\
\text { Drop(bar) }\end{array}$ & Coated/ & $\begin{array}{l}\text { Flow } \\
\text { Reynolds } \\
\text { Number }\end{array}$ & Specific Gravity & Buried/Surface & Compressibility Factor & \\
\hline 20 & coated & 4000 & 0.6657 & Buried & \\
\end{tabular}

Nigeria Gas Company (NGC) Eastern Division

Physical Configuration: From Warri to Okitipupa

Terrain transversed: From Ogharepe Warri to Okitipupa Ondo

Design Standard Code: ANSI/ASM E B31.8 Standard Code

\begin{tabular}{|l|l|l|l|l|l|l|}
\hline Length(km) & Diameter(cm) & Manifolds & $\begin{array}{l}\text { Design } \\
\text { Pressure(bar) }\end{array}$ & $\begin{array}{l}\text { Input/Output } \\
\text { Pressure(bar) }\end{array}$ & $\begin{array}{l}\text { Flow Rate }\left(\mathrm{m}^{3} / \mathrm{s}\right. \\
\text { Temperating } \\
\left({ }^{(0} \mathrm{C}\right)\end{array}$ & \\
\hline 122 & $36^{\prime \prime}(91.44 \mathrm{~cm})$ & 2 & 100 & $80.6 / 64$ & 1.8 & \\
\hline $\begin{array}{l}\text { Allowable } \\
\text { Pressure } \\
\text { Drop (bar) }\end{array}$ & Coated/ & $\begin{array}{l}\text { Flow } \\
\text { Reynolds } \\
\text { Number }\end{array}$ & Specific Gravity & Buried/Surface & Compressibility Factor & \\
\hline 16.6 & coated & 4000 & 1.326 & Buried & & \\
\hline
\end{tabular}

4000

Nigeria Gas Company (NGC) Western Division

Physical Configuration: From Okitipupa Ondo to Shagamu Lagos

Terrain transversed: From Okitipupa Ondo to Shagamu Lagos

Design Standard Code: ANSI/ASM E B31.8 Standard Code

\begin{tabular}{|c|c|c|c|c|c|c|}
\hline Length(km) & Diameter(cm) & Manifolds & $\begin{array}{l}\text { Design } \\
\text { Pressure(bar) }\end{array}$ & $\begin{array}{l}\text { Input/Output } \\
\text { Pressure(bar) }\end{array}$ & Flow Rate $\left(\mathrm{m}^{3} / \mathrm{s}\right.$ & $\begin{array}{l}\text { Operating } \\
\text { Temperature } \\
\left({ }^{10} \mathrm{C}\right)\end{array}$ \\
\hline 153 & $36^{\prime \prime}(91.44 \mathrm{~cm})$ & 2 & 100 & $64 / 44.4$ & 1.8 & 40 \\
\hline $\begin{array}{l}\text { Allowable } \\
\text { Pressure } \\
\text { Drop(bar) }\end{array}$ & $\begin{array}{l}\text { Coated/ } \\
\text { Uncoated }\end{array}$ & $\begin{array}{l}\text { Flow } \\
\text { Reynolds } \\
\text { Number }\end{array}$ & Specific Gravity & Buried/Surface & Compressibility Factor & \\
\hline 19.6 & coated & 4000 & 1.326 & Buried & 1.383 & \\
\hline
\end{tabular}

Table 1: Geometric, Configuration and Operational Data for the

$$
\text { Case Study Gas Pipelines }
$$

Source: Elftotal Nig. Ltd, Shell Petroleum Development Company, Agip Nig. Ltd, Nigeria Gas

Company (Eastern Division), Nigeria Gas Company (Western Division) 


\begin{tabular}{|c|c|c|c|c|c|c|c|c|c|c|}
\hline \multirow{2}{*}{\multicolumn{11}{|c|}{\begin{tabular}{l|l} 
C1 & C2 \\
ElfTotal Nig Ltd
\end{tabular}}} \\
\hline & & & & & & & & & & \\
\hline 0.84505 & 0.0427 & 0.025 & 0.01 & 0.009 & 0.007 & 0.006 & 0.0015 & 0 & 0 & 0.047 \\
\hline \multicolumn{11}{|c|}{ Shall Petroleum Development Company (SPDC) } \\
\hline 0.888 & 0.05423 & 0.0288 & 0.0072 & 0.009 & 0.0038 & 0 & 0.0018 & 0.00166 & 0.0002 & 0.0076 \\
\hline \multicolumn{11}{|c|}{ Agip Nig. Ltd } \\
\hline 0.8436 & 0.04116 & 0.02315 & 0.0165 & 0.0111 & 0.004 & 0.0033 & 0.0045 & 0.0033 & 0.00645 & 0.05 \\
\hline \multicolumn{11}{|c|}{ Nigeria Gas Company (Eastern Division) } \\
\hline 0.85 & 0.03795 & 0.02915 & 0.0155 & 0.0101 & 0.004 & 0.0033 & 0.00045 & 0.0003 & 0.00645 & 0.05 \\
\hline
\end{tabular}

Table 2: Gas Composition for the Case Study Pipelines

(Source: ElfTotal Nig. Ltd, Shell Petroleum Development Col '; PPmpany, Agip Nig. Ltd, Nigeria Gas

Company (Eastern Division), Nigeria Gas Company (Western Division))

The input data to the empirical scaling law model was derived from the optimal results of flow variables as in the previous works $[7,8]$ of the researcher. See Table 3.

\begin{tabular}{|c|c|c|c|}
\hline \multirow[t]{2}{*}{ Gas Company } & \multirow[t]{2}{*}{$\begin{array}{c}\text { Operational Throughput } \\
\mathbf{Q}_{\mathrm{opr}}\left(\mathrm{m}^{3} / \mathrm{s}\right)\end{array}$} & \multicolumn{2}{|c|}{$\begin{array}{c}\text { Optimal Throughput } \\
\mathbf{Q}_{\text {opt }}\left(\mathrm{m}^{3} / \mathrm{s}\right)\end{array}$} \\
\hline & & Pan-A & Pan-B \\
\hline ElfTotal Nig. Ltd & 1.8 & 1.89 & 2.8197 \\
\hline Shell Petroleum (SPDC) & 1.8 & 1.9423 & 3.3058 \\
\hline Agip Nig. Ltd & 1.8 & 1.8864 & 2.81932 \\
\hline $\begin{array}{c}\text { Nigeria Gas Company (NGC- } \\
\text { Eastern Division) }\end{array}$ & 1.8 & 1.9382 & 3.3008 \\
\hline
\end{tabular}

Table 3: Operational and Optimized Data

\section{Results and Discussion}

The percentage cost savings if the case study pipelines are operated optimally are as in Table 4 below:

\begin{tabular}{|c|c|c|c|}
\hline $\begin{array}{l}\text { PIPELINE } \\
\text { Designation }\end{array}$ & $\begin{array}{l}\text { OPERATING } \\
\text { CAPACITY } \\
\text { Q }_{\text {opr }}\left(\mathbf{m}^{3} / \mathbf{s}\right)\end{array}$ & $\begin{array}{l}\text { OPTIMAL } \\
\text { CAPACITY } \\
\text { Q }_{\text {opt }}\left(\mathrm{m}^{3} / \mathrm{s}\right)\end{array}$ & $\begin{array}{l}\text { Potential Cost Saving When } \\
\text { Operated Optimally }\end{array}$ \\
\hline \multicolumn{4}{|l|}{ ElfTotal } \\
\hline PANHANDLE A & $1.8 \mathrm{~m}^{3} / \mathrm{s}$ & $1.89 \mathrm{~m}^{3} / \mathrm{s}$ & $2.97 \%$ \\
\hline PANHANDLE B & $1.8 \mathrm{~m}^{3} / \mathrm{s}$ & $2.8197 \mathrm{~m}^{3} / \mathrm{s}$ & $30.91 \%$ \\
\hline \multicolumn{4}{|l|}{ Shell } \\
\hline PANHANDLE A & $1.8 \mathrm{~m}^{3} / \mathrm{s}$ & $1.9443 \mathrm{~m}^{3} / \mathrm{s}$ & $4.80 \%$ \\
\hline PANHANDLE B & $1.8 \mathrm{~m}^{3} / \mathrm{s}$ & $3.3058 \mathrm{~m}^{3} / \mathrm{s}$ & $44.00 \%$ \\
\hline \multicolumn{4}{|l|}{ Agip } \\
\hline PANHANDLE A & $1.8 \mathrm{~m}^{3} / \mathrm{s}$ & $1.8864 \mathrm{~m} 3 / \mathrm{s}$ & $2.85 \%$ \\
\hline PANHANDLE $\mathrm{B}$ & $1.8 \mathrm{~m}^{3} / \mathrm{s}$ & $3.3058 \mathrm{~m} 3 / \mathrm{s}$ & $44.01 \%$ \\
\hline \multicolumn{4}{|l|}{ NGC East } \\
\hline PANHANDLE A & $1.8 \mathrm{~m}^{3} / \mathrm{s}$ & $1.9382 \mathrm{~m} 3 / \mathrm{s}$ & $4.54 \%$ \\
\hline $\begin{array}{l}\text { PANHANDLE } \quad \mathrm{B} \\
\end{array}$ & $1.8 \mathrm{~m}^{3} / \mathrm{s}$ & $3.008 \mathrm{~m} 3 / \mathrm{s}$ & $36.08 \%$ \\
\hline \multicolumn{4}{|l|}{ NGCWest } \\
\hline PANHANDLE A & $1.8 \mathrm{~m}^{3} / \mathrm{s}$ & $1.9381 \mathrm{~m} 3 / \mathrm{s}$ & $4.54 \%$ \\
\hline PANHANDLE B & $1.8 \mathrm{~m}^{3} / \mathrm{s}$ & $3.005 \mathrm{~m} 3 / \mathrm{s}$ & $56.00 \%$ \\
\hline
\end{tabular}

Table 4: Output Results for the Economic Evaluation

In all cases optimized pipelines operating on Panhandle-A as the base equation witnessed $3 \%$ to S\% savings in installation cost. The margin of capital cost savings in investment cost was so tremendous for all the case study pipelines operating on optimized Modified Panhandle-B. The savings is to the tune of $30 \%$ to $60 \%$. 


\section{Recommendation for Future Research}

Installation costs of the five case study pipelines should be obtained from the five case pipelines to enable practical assessment of economic benefits of optimized Natural gas pipeline.

\section{Conclusion}

Economic benefits of gas pipeline flow optimization subject to flow throughput and pressure drop has been assessed. The margin of economic gain was substantial. This called for the review of the present operational conditions of our operating gas pipelines. The staggering economic margin necessitates the need to review the design, construction and operational procedures of our new generation gas pipeline network system.

\section{References}

i. Kohl, A. L. and Riesenfield, F. C. (1985): "Gas purification", 4thedition,

ii. Houston Gulf Publishing Company.

iii. Kohl, A. L. and Riesenfield, F. C. (1985): "Gas purification", 4thedition,

iv. Houston Gulf Publishing Company.

v. Cedigaz (2008): Cedigaz News Report, Vol. 4, No. 3, January 18, 2008.

vi. Cidigaz, (2009): "Natural Gas In The World", November 2009 edition, France.

vii. Dojey, A.; Jafferet, C.; Cornot-Gandolph, S.; Jullin, S and Valans, M. (1997):

viii. "Natural Gas Production, Processing and Transport", Edition Techrup, Paris,

ix. France.

x. Adeyanju, O. A. and Oyekunle, L. O. (2012): "Optimization of Natural Gas

xi. Transmission in Pipeline”, Oil and Gas Journal, Vol. 69, No 51.

xii. Shadrack, Mathew Uzoma, "Flow Optimization In Natural Gas Pipeline", PhD Thesis, April, 2016, Department of Mechanical Engineering, University of Port Harcourt, Port Harcourt, Rivers State, Nigeria.

xiii. Abam, D. P. S. (1997): Applied Engineering Management, $1^{\text {st }}$ Edition,

xiv. University Publication Limited, Lagos, Nigeria.

\section{Nomenclature}

Cost A-Capital cost of new facility being proposed

Cost B-Capital cost of existing similar facility

Size A- Capacity of facility A

Size B-Capacity of facility B

$\mathrm{N}-$ Empirical exponent, whose value is between 0.4 and 0.8 ; for facilities laid in

$\mathrm{Q}_{\text {opt }}$-Optimal flow capacity

$\mathrm{Q}_{\text {opr}}$-Operational flow capacity 